American Journal of Applied Sciences 6 (1): 89-92, 2009

ISSN 1546-9239

(C) 2009 Science Publications

\title{
RBFNN Model for Predicting Nonlinear Response of Uniformly Loaded Paddle Cantilever
}

\author{
Abdullah H. Abdullah \\ Department of Electromechanical Engineering, \\ University of Technology, Baghdad, Iraq
}

\begin{abstract}
The Radial basis Function neural network (RBFNN) model has been developed for the prediction of nonlinear response for paddle Cantilever with built-in edges and different sizes, thickness and uniform loads. Learning data was performed by using a nonlinear finite element program, incremental stages of the nonlinear finite element analysis were generated by using 25 schemes of built paddle Cantilevers with different thickness and uniform distributed loads. The neural network model has 5 input nodes representing the uniform distributed load and paddle size, length, width and thickness, eight nodes at hidden layer and one output node representing the max. deflection response $(1500 \times 1$ represent the deflection response of load). Regression analysis between finite element results and values predicted by the neural network model shows the least error.
\end{abstract}

Key words: RBFNN, cantilever, finite element, ANSYS

\section{INTRODUCTION}

Modeling and simulation are indispensable when dealing with complex engineering systems. It makes it possible to do essential assessment before systems are built, it can alleviate the need for expensive experiments and it can provide support in all stages of a project from conceptual design, through commissioning and operation. Cantilever sensors are the most important electric machinery in all the fields of industry. Cantilever sensors are based on relatively well known and simple transduction principle. A simple cantilever beam can be used as a sensor for biomedical, chemical and environmental applications. When microfabricated multilayered cantilever beam is exposed to sensing environment, it bends because of single or a combination of external forces like electrostatic, electric, magnetic, mass, nuclear radiation or mere mass. Similarly, it can bend because of intrinsic stresses generated due to chemical, physical or thermal means within the upper layer of cantilever itself.

As recent research efforts advance in several converging areas of science and technology, cantileverbased sensors have been proved to be quite versatile and sensitive devices and have been used mainly in the trace detection of bio-chemical materials. The cantilever method of bio-chemical sensing does not require any fluorescence tagging, therefore gets many attentions $^{[1,2]}$. Micromachined silicon cantilever beams have been applied in fluid flow volume sensing ${ }^{[3,4]}$.
In addition, the actual mechanism for detection of the cantilever deflections is also very important. The amount of deflections of a cantilever beam can be detected by several read-out systems, including optical detection, capacitive detection, tunneling detection and interferometer detection. The optical level technique and the piezoresistive method are usually used to detect cantilever beam deflection. In general, the deflection is caused by its interaction with measured under circumstances of stress, a small force and a change of mass or temperature. However, for more complex structures, finite element modeling is useful to analyze and optimize these structures ${ }^{[5]}$.

This research the present work will explore the use of Redial Base Function Neural Network (RBFNN) modeling of the paddle cantilevers in conjugation with finite element analysis (FEA) software. The model is constructed through the use of the neural network design (nntool) toolbox in MATLAB.

\section{THEORETICAL MODEL ANALYSIS}

In this section, the simple and paddle cantilever sensors as shown in Fig. 1-2 were modeled using the static equations of mechanics. To calculate the amount of deflection at the tip of a cantilever beam, the differential equation of a cantilever beam for a small deflection is given by ${ }^{[6]}$.

$$
E I \frac{d^{2} y(x)}{d x^{2}}=M
$$



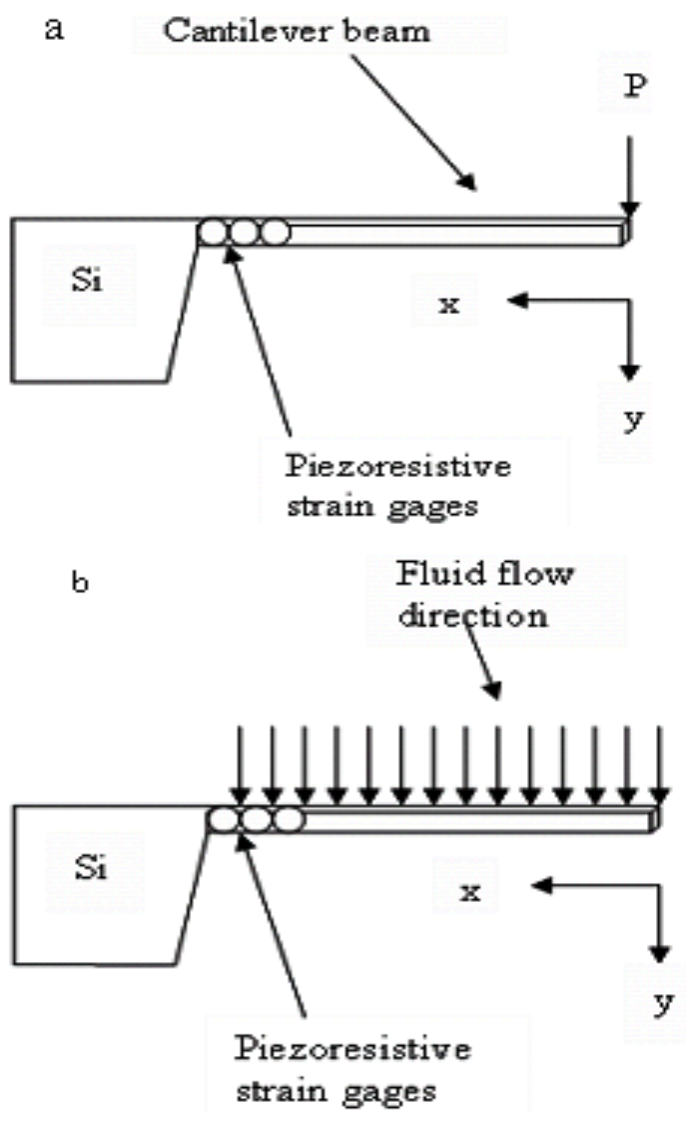

Fig.1: Simple cantilever, a: Single force applied at the free end, b: Uniformly distribute force a long the beam

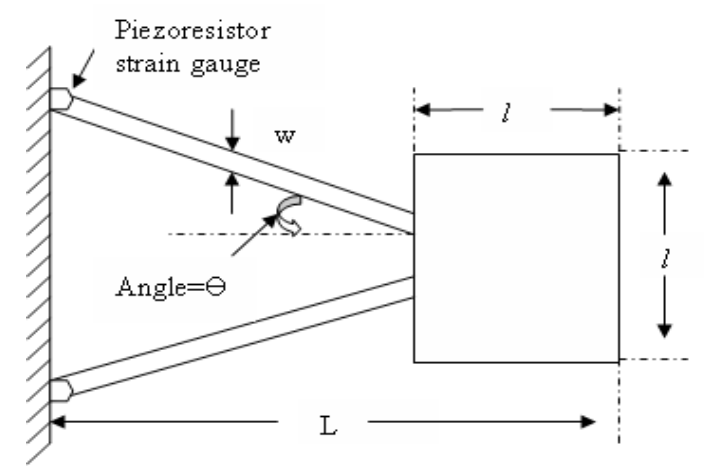

Fig. 2: Paddle cantilever

where $\mathrm{M}$ is the bending moment, $\mathrm{E}$ is Young's modulus, $\mathrm{y}(\mathrm{x})$ is the deflection along the cantilever beam and I is the area moment of the cross section with respect to the neutral axis of the cantilever. $\mathrm{M}=\mathrm{Px}$ when a single force $\mathrm{P}$ is applied on the free end of the cantilever. $\mathrm{M}=\mathrm{qx}^{2} / 2$ under a flowing fluid situation, where $\mathrm{q}$ is a force element at the position $\mathrm{x}$ along the cantilever beam and is proportional to the surface area facing towards the flowing fluid and drag force. The drag force is proportional to the fluid density, the drag coefficient of the cantilever and the flow velocity squared in a turbulent flow or flow velocity in a laminar flow.

When the $\mathrm{x}$-axis origin is selected at the free end of the cantilever beam, the boundary conditions are given by

$$
\begin{array}{ll}
\mathrm{x}=\mathrm{L} \quad \frac{\mathrm{dy}}{\mathrm{dx}}=0 \\
\mathrm{x}=\mathrm{L} \text { then } \mathrm{y}=0
\end{array}
$$

Now we integrate the differential equation for cantilever deflection and use the above mentioned boundary conditions.

$$
\frac{\mathrm{dy}(\mathrm{x})}{\mathrm{dx}}=\frac{1}{\mathrm{EI}}\left[\mathrm{Px}^{2} / 2+\mathrm{K}_{1}\right]
$$

Eventually, the deflection of the cantilever beam when a single force is applied at the free end of the cantilever is given as:

$$
y(x)=\frac{2 P}{E w t^{3}}\left[x^{3}-3 L^{2} x+2 L^{3}\right]
$$

\section{RBFNN FOR PADDLE CANTILEVER DEFLECTION}

From the examples ANN captures the domain knowledge. ANN can handle continuous as well as discrete data and have good generalization capability as with fuzzy expert systems. An ANN is a computational model of the brain. They assume that computation is distributed over several simple units called neurons, which are interconnected and operate in parallel thus known as parallel distributed processing systems or connectionist systems. Implicit knowledge is built into a neural network by training it. Several types of ANN structures and training algorithms have been proposed.

The basic form of RBF architecture involves entirely three different layers. The input layers is made $\mathrm{n}$, of source nodes while the second layer is hidden layer of high enough dimension which senses a different purpose from that in a multilayer perception. The output layer supplies the response of the network to the activation patterns applied to the input layer. The tram formation from the input layer to hidden is nonlinear whereas the transformation from the hidden from unit to the output layer is linear. 


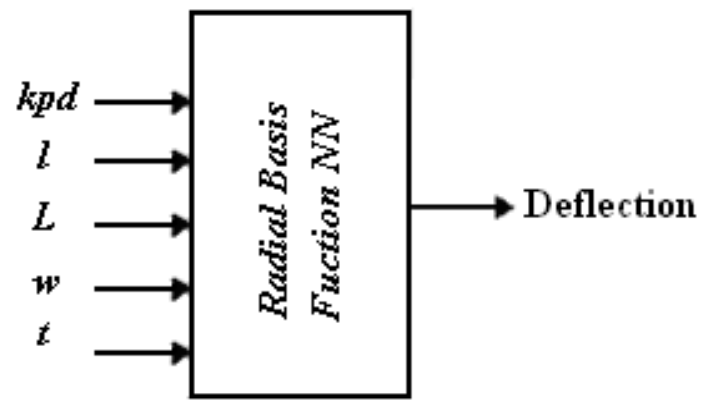

Fig. 3: RBFANN for paddle cantilever

The transfer function for a radial basis neuron is:

$$
\operatorname{radbas}(n)=e^{-n 2}
$$

This function calculates a layer's output from its net input.

For effective predicting of paddle cantilever, the selection of proper inputs and outputs of ANN, structure of the network and training of it using appropriate data should be done with utmost care. In the present study, inputs are selected as uniform distributed load, paddle cantilever size, length, width and thickness. The NN outputs have been termed as one output node representing the max. deflection response $(1500 \times 1$ represent the deflection response of load) Fig. 3.

\section{COMPUTER MODELING}

The cantilever beam was modeled using ANSYS software. Figure 4 is screen snapshot of how ANSYS modeled the paddle cantilever beam. Modeling begins by choosing an element type, a beam, truss element, frame, solid, shell, etc. Solid 92 was chosen as the element type because it was a three-dimensional element and had the capability of six degrees-ofmovement at each node point. Node locations were entered in Cartesian coordinates and these were used to construct an area and then a volume. The volume was then meshed, which fills in node points throughout the entire volume for the finite element analysis. Before a solution is run, loading of the beam which includes both constraints and actual loads must be added. In Fig. 3, the left side two ends of paddle cantilever of the beam were completely restrained in all degrees of freedom. A gravitational force was applied and then the solution was implemented. Figure 5 shows the structure in its deformed and unreformed shape after analysis. The maximum deflection of the beam occurred at the beam tip.

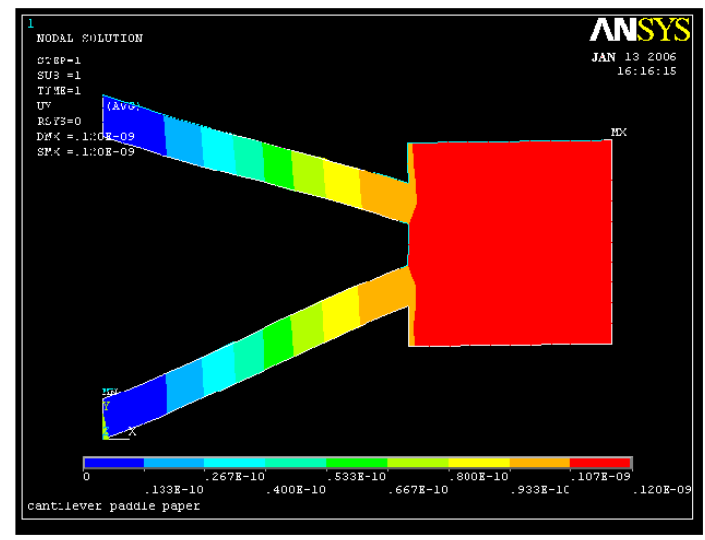

Fig. 4: Shows the structure in its deformed shape after analysis

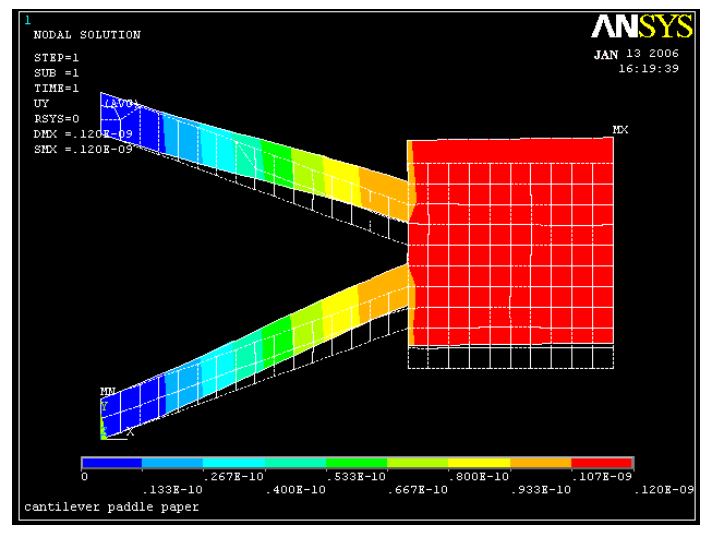

Fig. 5: Shows the structure in its deformed and unreformed shape after analysis

Using this ANSYS software the simple and paddle cantilever were modeled for a series of structures and found the sensitivities and also compared with theoretical calculated sensitivities, this will be covered in detail in analysis and results section.

\section{ANALYSIS AND RESULTS}

To train ANN models with the results of the finite element analyses, network architecture was required; first the entire training data file was randomly divided into training and testing data sets. About $90 \%$ of the data 1350 patterns, were used to train the different network architectures where remaining 150 patterns were used for testing to verify the prediction ability of each trained NN model. Since RBFNNs Learn relations and approximate function mapping limited by the extent of the training data, the best use of the trained RBFNN models can be achieved in interpolation. 
Am. J. Applied Sci., 6 (1): 89-92, 2009

Table1: Max. Deflection for a Set of Paddle Cantilever Sensors and Dimensions $\left(10^{-6} \mathrm{~m}\right)$

\begin{tabular}{|c|c|c|c|c|c|}
\hline $\mathrm{L}$ & w & $\mathrm{t}$ & $\begin{array}{l}\mathrm{Ksp} \\
\left(\mathrm{Nm}^{-1}\right)\end{array}$ & $\begin{array}{l}\text { Vertical } \\
\text { Deflection } \\
\text { FEM }\end{array}$ & $\begin{array}{l}\text { Vertical } \\
\text { Deflection } \\
\text { RBFNN }\end{array}$ \\
\hline 200 & 25 & 2.5 & 1.831 & 5.933 & 5.822 \\
\hline 250 & 25 & 2.5 & 0.938 & 3.848 & 3.721 \\
\hline 300 & 25 & 2.5 & 0.5425 & 2.695 & 2.562 \\
\hline 350 & 25 & 2.5 & 0.3417 & 1.993 & 1.926 \\
\hline 300 & 30 & 2.5 & 0.6510 & 2.695 & 2.599 \\
\hline 350 & 30 & 2.5 & 0.4100 & 1.993 & 1.901 \\
\hline 400 & 30 & 2.5 & 0.2747 & 1.533 & 1.492 \\
\hline 450 & 30 & 2.5 & 0.1929 & 1.215 & 1.197 \\
\hline 400 & 40 & 2.5 & 0.3662 & 1.533 & 1.487 \\
\hline 450 & 40 & 2.5 & 0.2572 & 1.215 & 1.197 \\
\hline 500 & 40 & 2.5 & 0.1875 & 0.987 & 1.001 \\
\hline 550 & 40 & 2.5 & 0.1409 & 0.818 & 0.795 \\
\hline
\end{tabular}

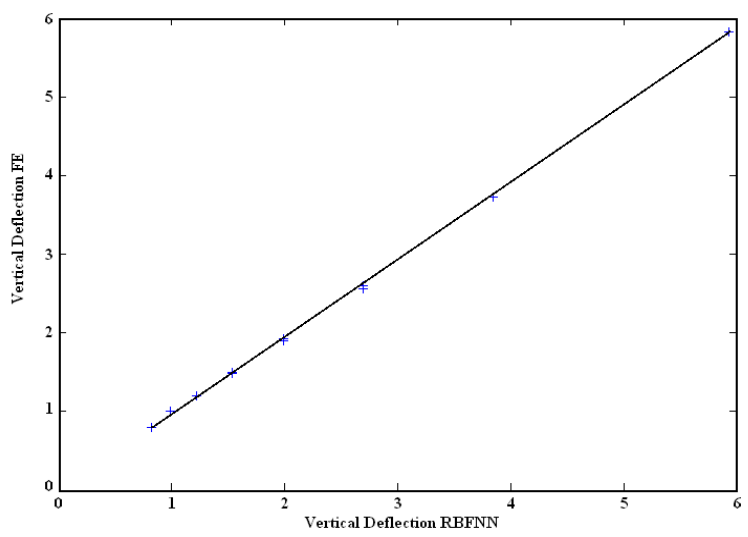

Fig. 6: Shows maximum deflections by finite element against corresponding RBFNN prediction

Table 1 shows comparison sample of max. deflection for a set of paddle cantilever sensors has been calculated by RBFNN and FEM techniques. The Young's modulus E coefficient of silicon is taken as $1.5^{*} 10^{11} \mathrm{~N} \mathrm{~m}^{-2}$ in our design.

From the analysis of the results in Table 1, it is observed that the accuracy of the RBFNN method was slightly superior when compared to the FEM techniques on account of mean average error (MAE). Figure 6 shows a plot of finite element maximum deflections against corresponding ANN prediction. A linear correlation can be observed and the correlation coefficient was found Table 1 .

The computational time is the least, for the (ANN) prediction is much less as compared with the FEM, from Table 1 we can see that the computational time for
ANN after training is $0.0016 \mathrm{sec}$ which is less than that for FEM $(1.83 \mathrm{sec})$, it means RBFNN can often obtain results in almost negligible time as compared to similar works using the FE methods. The efficiency of RBFNN depends upon its accuracy, speed and memory requirements.

\section{CONCLUSIONS}

In the present study the Radial Basis Function (RBFNN) has been explored for predicting nonlinear response of uniformly loaded paddle cantilever. The simulation data from ANSYS software has been used for training and testing. The Simulation results show that RBF can be very successively used for reduction of the effort and time required determining the loaddeflection response of paddle cantilever as the FE methods usually deal with only a single problem for each run. This means that it can solve many problems that have mathematical and time difficulties.

\section{REFERENCES}

1. Lang, H.P., M.K. Baller, F.M. Battiston, J. Fritz, R. Berger, J.P. Ramseyer, P. Fornaro, E. Meyer, H.J. Güntherodt, J. Brugger, U. Drechsler, H. Rothuizen, M. Despont, P. Vettiger, Ch. Gerber and J.K. Gimzewski, 1999. The nanomechanical NOSE, IEEE Proceedings MEMS'99, Orlando, Florida, USA, pp: 9-13.

2. Boisen, A., J. Thaysen, H. Jensenius and O. Hansen, 2000. Environmental sensors based on micromachined cantilevers with integrated readout. Ultramicroscopy, 82: 11.

3. Gupta, D.A. and R. Bashir, 2004. Single virus particle mass detection using microresonators with nanoscale thickness. Applied Phys. Lett., 84 (11): 1976-1978.

4. Ilic, B., Y. Yang and H.G. Craighead, 2004. Virus detection using nano electromechanical devices. Applied Phys. Lett., 85 (13): 2604-2606.

5. Gass, V., B.H. van der Schoot and N.F. de Rooij, 1993. Nanofluid handling by micro-flow sensor based on drag force measurement. Proceeding MEMS 93 (New York: IEEE), pp: 167-72.

6. Timoshenko S. Strength of Materials, Part 1 Elementary Theory and Problems. 3rd Edn., (Princeton, NJ: Van No strand), pp: 137-40. 